\title{
EVALUACIÓN MEDIANTE ELECTRORRETINOGRAFÍA DE LA TOXICIDAD DEL AZUL TRIPÁN EN LA CIRUGÍA DEL AGUJERO MACULAR
}

\section{EVALUATION OF TRYPAN-BLUE TOXICITY IN MACULAR HOLE SURGERY WITH ELECTRORETINOGRAPHY}

\author{
FIGUEROA MS ${ }^{1}$, REBOLLEDA $\mathrm{G}^{1}$, NOVAL $\mathrm{S}^{1}$, CONTRERAS I ${ }^{1}$
}

\begin{abstract}
RESUMEN
Objetivo: Existen dudas sobre la posible toxicidad retiniana de los colorantes vitales. Se diseñó un estudio prospectivo para determinar la posible toxicidad del azul tripán (AT) en la cirugía del agujero macular (AM) con pelado de la membrana limitante interna (MLI) tras su tinción con AT mediante la realización de electrorretinogramas (ERG).

Métodos: Pacientes con AM fueron evaluados antes y 6 meses después de la cirugía. A todos se les realizó una vitrectomía con tinción de la MLI bajo aire. Los pacientes fáquicos fueron sometidos simultáneamente a una facoemulsificación con implante de lente intraocular. Se crearon dos grupos de estudio: en el grupo 1, la tinción de la MLI se realizó con AT al 0,06\% y en el grupo 2 con AT al $0,15 \%$. El ERG preoperatorio se realizó en la semana previa a la cirugía, el postoperatorio entre 3 y 6 meses después. Se procedió a la comparación de los datos del ERG entre el ojo con afecto y el adelfo en las fases pre- y postoperatoria. También se evaluaron los cambios en agudeza visual (AV) en ambos grupos.
\end{abstract}

Resultados: Se reclutaron nueve pacientes en cada uno de los grupos. La AV mejoró de forma signifi-

\begin{abstract}
Purpose: Concern has been raised about the retinal toxicity of vital dyes. We designed a prospective study to determine the possible toxicity of trypanblue (TB) in macular hole surgery with TB-assisted internal limiting membrane (ILM) peeling through the performance of electroretinograms (ERGs).

Methods: Patients diagnosed with a macular hole underwent ophthalmological evaluation prior to surgery and at 6 months follow-up. All patients underwent vitrectomy and ILM-staining under air. All phakic patients underwent phacoemulsification and IOL implantation simultaneously. There were two study groups: In group 1, ILM-staining was performed with $0.06 \% \mathrm{~TB}$, while in group 2 the procedure was performed with $0.15 \%$ TB. Preoperative ERG recordings were measured in the week prior to surgery. Postoperative ERGs were measured 3 to 6 months after surgery. The ERG data between eyes with macular hole and fellow eyes were compared in the pre- and post-operative stages. Visual acuity (VA) changes in both groups were evaluated.
\end{abstract}

Results: Nine patients were recruited in each group. VA improved significantly in both groups,

Recibido: 25/10/07. Aceptado: 28/10/08.

Servicio de Oftalmología. Hospital Universitario Ramón y Cajal. Madrid. España.

1 Doctor en Medicina.

Correspondencia:

Inés Contreras Martín

C/. Martín de los Heros, 8/0 5. ${ }^{\circ} \mathrm{D}$

28008 Madrid

España

E-mail: inescon3@yahoo.com 
cativa en ambos grupos: más de un $65 \%$ de los pacientes mejoró más de dos líneas. No existían diferencias en mejoría de AV entre ambos grupos ni en los parámetros del ERG entre el ojo afecto y el adelfo.

Conclusión: No se detectaron signos de toxicidad retiniana tras la tinción con AT.

Palabras clave: Electrorretinografía, cirugía del agujero macular, colorantes vitales, efectos secundarios, toxicidad por azul tripán. with more than $65 \%$ of patients improving more than 2 lines. There were no statistical differences in VA gain between groups or in ERG values between affected and fellow eyes.

Conclusion: No significant retinal toxicity of TB staining could be clinically detected (Arch Soc Esp Oftalmol 2008; 83: 659-664).

Key words: Electroretinography, macular hole surgery, side-effects, trypan-blue toxicity, vital dyes.

\section{INTRODUCCIÓN}

Diversos estudios han descrito la utilidad del pelado de la membrana limitante interna (MLI) en la cirugía del agujero macular (1-3). Dada la dificultad técnica de esta maniobra, se han desarrollado colorantes vitales que ayuden al cirujano. Así, el verde de indocianina (VI) tiñe la MLI in vivo y facilita su extracción, habiéndose obtenido elevadas tasas de cierre anatómico del agujero macular (4, 5). Sin embargo, se han descrito casos de daño funcional y anatómico tras la tinción de la MLI con VI (6-10).

El azul tripan (AT) es un colorante vital que produce una tinción más tenue que la obtenida con VI, que sin embargo resulta suficiente para permitir su identificación durante la maniobra de pelado. Diversos grupos han obtenido buenos resultados funcionales tras la vitrectomía con pelado de la MLI con tinción con AT, incluyendo mejorías significativas de agudeza visual (AV) sin defectos perimétricos detectables $(11,12)$.

Tras la publicación de varios trabajos que generaban dudas sobre la posible toxicidad del AT (13, 14), decidimos realizar un estudio prospectivo en pacientes con agujero macular para determinar si el AT puede ser tóxico para la retina, mediante la realización de electroretinogramas (ERG) antes y después de la cirugía. Resultados preliminares realizados con AT al 0,06\% no mostraron cambios significativos en el ERG con respecto al ojo adelfo (15). Dado que actualmente se están empleando concentraciones mayores de AT, se amplió el estudio para estudiar la seguridad del pelado de la MLI tras tinción con AT al 0,15\% en el tratamiento del agujero macular.

\section{SUJETOS, MATERIAL Y MÉTODOS}

Se diseñó un estudio prospectivo intervencional no randomizado, con pacientes diagnosticados de agujero macular en estadio 2 a 4 según la clasificación de Gass, de menos de 1 año de evolución. Los criterios de exclusión fueron la presencia de otras patologías oculares que pudiesen producir alteraciones en el ERG, excepto la opacidad cristaliniana, tanto en el ojo afecto como en el adelfo.

Todos los pacientes fueron sometidos a una exploración oftalmológica completa previa a la cirugía, incluyendo agudeza visual (AV) y biomicroscopía de los segmentos anterior y posterior.

A todos los pacientes se les realizó una vitrectomía vía pars plana bajo anestesia retrobulbar. Una vez retirada la hialoides posterior, se realizó un intercambio fluido-aire. La membrana limitante interna se tiñó sobrepasando las arcadas temporales con $0,1 \mathrm{ml}$ de AT durante 2 minutos. Se emplearon pinzas de Tano asimétricas para el pelado de la MLI. Al final de la cirugía, se introdujo $\mathrm{C}_{3} \mathrm{~F}_{8}$ al $8 \%$ en la cavidad vítrea, indicando a los pacientes que mantuviesen una postura con la cara hacia abajo durante 2 semanas. El estudio se realizó en dos etapas. En la primera, se empleó AT al 0,06\% para teñir la MLI (Grupo 1) y en la segunda, AT al 0,15\% (Grupo 2). En todos los pacientes fáquicos se realizó un procedimiento de facoemulsificación con implante de lente intraocular en el mismo acto quirúrgico que la vitrectomía.

Los pacientes fueron evaluados en los días 1 y 7 y en los meses 1,3 y 6 tras la cirugía. El agujero macular se consideró cerrado si había desaparecido completamente en el examen con lámpara de hendidura, siendo la prueba de Watzke-Allen negativa. 
En caso contrario, se clasificó como abierto, de acuerdo con los criterios del grupo de estudio del agujero macular del Moorfields (16).

El ERG preoperatorio se realizó en la semana previa a la cirugía. El ERG postoperatorio se realizó entre 3 y 6 meses tras la vitrectomía. El ERG de campo completo (registro de bastones, combinado de conos y bastones, de conos, potenciales oscilatorios y parpadeo [flicker] a $30 \mathrm{~Hz}$ ) se realizó de acuerdo con los criterios de la International Society for Clinical Electrophisiology of Vision (ISCEV) empleando un sistema de electrodiagnóstico controlado por ordenador PRIMUS 2,5 (17). Se compararon los datos del ERG del ojo con agujero macular y el ojo adelfo antes y después de la cirugía.

Se emplearon pruebas no paramétricas para el análisis estadístico. La relación entre la AV pre- y postoperatoria se analizó mediante el test de Wilcoxon para muestras pareadas. Los valores del ERG del ojo afecto y adelfo se compararon mediante la prueba de Mann-Whitney.

El análisis estadístico se realizó con el programa SPSS 8.0 (SPSS Inc., Chicago, Illinois, USA).

\section{RESULTADOS}

Se incluyeron nueve pacientes en cada uno de los grupos. Los datos de los pacientes se recogen en la tabla I. El agujero macular se cerró tras la cirugía en ocho pacientes del grupo $1(88,9 \%)$ y en todos los pacientes del grupo 2 , sin que esta diferencia fuese estadísticamente significativa. La AV media preoperatoria era de 0,08 en el grupo 1 (desviación estándar [DE] 0,06) y 0,09 en el grupo 2 (DE 0,07). En ambos grupos la AV mejoró significativamente en el postoperatorio, a 0,38 en el grupo 1 (DE 0,23) y a 0,35 en el grupo 2 (DE 0,15), p=0,012 para ambos grupos. No se detectaron diferencias significativas en la AV pre- ni postquirúrgica entre ambos grupos. La AV mejoró en dos o más líneas en siete de nueve pacientes del grupo $1(77,8 \%)$ y en seis de nueve pacientes del grupo $2(66,7 \%)$.

Los valores del ERG se muestran en las tablas II y III. No existían diferencias significativas entre los ojos con agujero macular y los ojos adelfos ni antes ni después de la cirugía.

\section{DISCUSIÓN}

Los colorantes vitales se han empleado en la cirugía del agujero macular para teñir la MLI debido a la dificultad que entraña su identificación y extracción completa. El verde indiocianina fue el colorante más utilizado inicialmente y se han publicado resultados anatómicos y funcionales positivos $(4,5)$. Sin embargo, diversos autores han descrito la apari-

Tabla I. Datos basales y resultados quirúrgicos en los dos grupos de estudio

\begin{tabular}{lclcccc}
\hline Paciente & Edad (años) & Sexo & Estadio AM & AV preoperatoria & AV postoperatoria & Resultado anatómico \\
\hline Grupo 1 (0,06\%) & & & & & \\
1 & 69 & Mujer & 4 & 0,02 & 0,1 & Cerrado \\
2 & 69 & Hombre & 4 & 0,1 & Cerrado \\
3 & 84 & Hombre & 3 & 0,1 & Cerrado \\
4 & 67 & Mujer & 2 & 0,2 & 0,8 & Cerrado \\
5 & 71 & Mujer & 3 & 0,1 & 0,5 & Cerrado \\
6 & 72 & Mujer & 2 & 0,02 & 0,5 & Cerrado \\
7 & 66 & Mujer & 3 & 0,02 & 0,4 & Cerrado \\
8 & 72 & Hombre & 3 & 0,1 & 0,3 & Cerrado \\
9 & 69 & Mujer & 3 & 0,02 & 0,02 & Abierto \\
Grupo 2 (0,15\%) & & & & 0,1 & 0,3 & Cerrado \\
1 & 64 & Mujer & 3 & 0,02 & 0,5 & Cerrado \\
2 & 61 & Mujer & 3 & Contar dedos & 0,02 & Cerrado \\
3 & 71 & Hombre & 2 & 0,02 & 0,4 & Cerrado \\
4 & 71 & Mujer & 3 & 0,15 & 0,3 & Cerrado \\
5 & 81 & Mujer & 4 & 0,2 & 0,5 & Cerrado \\
6 & 70 & Mujer & 2 & 0,1 & 0,3 & Cerrado \\
7 & 72 & Hombre & 4 & 0,2 & 0,4 & Cerrado \\
9 & 61 & Hombre & 4 & 3 & 0,05 & Cerrado \\
\hline \hline
\end{tabular}

AM: agujero macular; AV: agudeza visual. 
Tabla II. Valores del ERG pre- y postoperatorio en el grupo 1 (Azul tripán al 0,06\%) en ojos con agujero macular (AM) y ojos adelfos (control)

\begin{tabular}{|c|c|c|c|c|c|c|c|c|c|c|c|c|}
\hline \multirow[b]{3}{*}{ Grupo $1(0,06 \%)$} & \multicolumn{6}{|c|}{ Preoperatorio } & \multicolumn{6}{|c|}{ Postoperatorio } \\
\hline & \multicolumn{3}{|c|}{ Amplitud } & \multicolumn{3}{|c|}{ Tiempo implícito (ms) } & \multicolumn{3}{|c|}{ Amplitud } & \multicolumn{3}{|c|}{ Tiempo implícito (ms) } \\
\hline & $\mathrm{AM}$ & Control & $\mathrm{p}$ & $\mathrm{AM}$ & Control & $\mathrm{p}$ & $\mathrm{AM}$ & Control & $\mathrm{p}$ & $\mathrm{AM}$ & Control & $\mathrm{p}$ \\
\hline Bastones & 154,83 & 177,9 & 0,400 & 103,21 & 101,2 & 0,628 & 174,81 & 175,26 & 0,989 & 98,26 & 97,82 & 0,942 \\
\hline Combinado conos-bastones & & & & & & & & & & & & \\
\hline onda a & 238,9 & 249,37 & 0,674 & 24,15 & 23,77 & 0,542 & 209,36 & 225,53 & 0,501 & 24,76 & 23,60 & 0,271 \\
\hline onda $\mathrm{b}$ & 490,52 & 496,25 & 0,850 & 47,46 & 53,71 & 0,274 & 488,41 & 524,00 & 0,435 & 47,92 & 46,96 & 0,796 \\
\hline OPsP2 & 68,82 & 79,87 & 0,253 & 26,20 & 25,94 & 0,658 & 73,47 & 83,15 & 0,652 & 26,70 & 26,05 & 0,431 \\
\hline Conos & 129,30 & 134,63 & 0,811 & 30,67 & 29,58 & 0,147 & 151,83 & 130,71 & 0,435 & 30,84 & 30,62 & 0,773 \\
\hline $30 \mathrm{~Hz}$-flicker & 101,75 & 118,70 & 0,266 & 27,43 & 28,72 & 0,352 & 104,00 & 104,00 & 0,980 & 28,67 & 26,50 & 0,239 \\
\hline
\end{tabular}

Tabla III. Valores del ERG pre- y postoperatorio en el grupo 1 (Azul tripán al 0,15\%) en ojos con agujero macular (AM) y ojos adelfos (control)

\begin{tabular}{|c|c|c|c|c|c|c|c|c|c|c|c|c|}
\hline \multirow[b]{3}{*}{ Grupo $2(0,15 \%)$} & \multicolumn{6}{|c|}{ Preoperatorio } & \multicolumn{6}{|c|}{ Postoperatorio } \\
\hline & \multicolumn{3}{|c|}{ Amplitud } & \multicolumn{3}{|c|}{ Tiempo implícito (ms) } & \multicolumn{3}{|c|}{ Amplitud } & \multicolumn{3}{|c|}{ Tiempo implícito (ms) } \\
\hline & $\mathrm{AM}$ & Control & $\mathrm{p}$ & $\mathrm{AM}$ & Control & $\mathrm{p}$ & $\mathrm{AM}$ & Control & $\mathrm{p}$ & $\mathrm{AM}$ & Control & $\mathrm{p}$ \\
\hline Bastones & 152,20 & 137,3 & 0,50 & 88,30 & 90,4 & 0,50 & 126,3 & 118,4 & 0,84 & 92,26 & 97,4 & 0,63 \\
\hline \multicolumn{13}{|c|}{ Combinado conos-bastones } \\
\hline onda a & 182,90 & 143,7 & 0,14 & 24,16 & 25,5 & 0,91 & 191,2 & 163,3 & 0,48 & 25,20 & 25,3 & 1 \\
\hline onda $\mathrm{b}$ & 436,11 & 395,9 & 0,46 & 46,68 & 47,5 & 0,67 & 445,8 & 415,7 & 0,84 & 50,60 & 49,6 & 0,52 \\
\hline OPsP2 & 51,34 & 51,7 & 0,67 & 27,70 & 28,7 & 0,49 & 60,9 & 54,6 & 0,52 & 27,80 & 28,4 & 0,91 \\
\hline Conos & 101,49 & 91,2 & 0,69 & 30,40 & 31,6 & 0,33 & 112,9 & 91,8 & 0,27 & 32,50 & 31,9 & 0,56 \\
\hline $30 \mathrm{~Hz}$-flicker & 73,10 & 68,6 & 0,86 & 28,14 & 26,6 & 0,48 & 82,8 & 73,5 & 0,52 & 27,90 & 28,4 & 0,74 \\
\hline
\end{tabular}

ción de efectos adversos posiblemente relacionados con el uso del VI y que dieron lugar a resultados visuales desfavorables $(7,8)$. Se han documentado defectos campimétricos permanentes incluso con concentraciones del $0,5 \%$ y tiempos de exposición de menos de un minuto (8-10), así como el desarrollo de atrofia del nervio óptico $(9,10)$. También se ha relacionado la atrofia del epitelio pigmentario de la retina con la ausencia de mejoría visual tras la cirugía (7).

El azul tripán es un colorante vital con menor difusión que el VI debido a que produce tan sólo una tinción débil de la MLI a la concentración disponible (Vision Blue ${ }^{\circledR}, 0,06 \%$ ). Sin embargo, si se inyecta bajo aire, la tinción de la MLI mejora. La nueva presentación comercial, Membrane Blue ${ }^{\circledR}$, al $0,15 \%$, permite obtener una mejor tinción. Este estudio fue realizado en dos etapas para comparar la relación riesgo/beneficio de las distintas concentraciones de AT: en la primera, se empleó AT al 0,06\% para facilitar la eliminación de la MLI, en la segunda la concentración fue de $0,15 \%$.
Estudios experimentales no han detectado daños en las células del EPR tras la utilización de AT, a diferencia de la toxicidad tiempo y dosis dependiente del VI (18). Se han comunicado resultados visuales favorables tras la cirugía del agujero macular con pelado de MLI con AT, con mejoría de la AV y la ausencia de defectos campimétricos. No se han descrito cambios en el aspecto funduscópico $(11,12)$ aunque algunos estudios han apuntado a una posible toxicidad sobre la retina neurosensorial $(13,14)$.

Se ha demostrado que la vitrectomía con C3F8 acelera la formación de cataratas $(1,2)$. Además, la opacificación cristaliniana reduce la amplitud de las ondas a y b del ERG y prolonga los tiempos implícitos como consecuencia de la reducción de la luz que alcanza los fotorreceptores. Por todo ello, se decidió realizar de forma simultánea a la vitrectomía una facoemulsificación con implante de LIO a todos los pacientes fáquicos.

La ERG se ha utilizado en estudios animales para detectar los posibles efectos tóxicos de los colorantes vitales. Así, Veckeneer y colaboradores no detec- 
taron cambios en los principales valores del ERG en ojos de conejo tras la exposición al AT (19).

En el ERG patrón, el componente P50, empleado para evaluar la función macular, evoluciona de forma paralela a la AV tras la cirugía, lo que podría dificultar la detección de daño macular. Por esta razón se decidió realizar este estudio con ERG de campo completo. Este tipo de ERG no muestra alteraciones en presencia de lesiones maculares focales porque la mácula representa solamente un $10 \%$ de las respuestas. Por esta razón, no existían diferencias en el ERG preoperatorio entre los ojos con AM y los adelfos. Sin embargo, si durante la cirugía se extiende el colorante más allá de la mácula, el ERG de campo completo podría resultar útil para evaluar la toxicidad del AT. En este estudio, los valores postoperatorios del ERG no mostraron diferencias entre el ojo con AM y el control, lo que parece indicar que el AT es bien tolerado y seguro para la tinción de la MLI.

Somos conscientes de que el ERG multifocal podría ser más apropiado para evaluar y cuantificar la toxicidad macular focal. Sin embargo, el ERG multifocal también evoluciona en paralelo con la AV. Un artículo reciente que evaluaba la toxicidad del AT al $0,15 \%$ en pacientes con membranas epirretinianas no detectó ningún signo de daño tras la vitrectomía (20).

En nuestro estudio, los resultados anatómicos y visuales fueron favorables. El porcentaje de cierre anatómico (89 y 100\%) es similar al obtenido por otros grupos. A pesar de emplear un colorante vital, la AV mejoró significativamente en ambos grupos, sin que se pudiesen detectar diferencias en el resultado visual en función de la concentración del AT. La intensidad de la tinción con AT se puede aumentar al realizar la tinción bajo aire o bien diluyendo el AT en glucosa al 5\%. Actualmente estamos investigando la utilidad de este último método para reducir las maniobras quirúrgicas. Recientemente se han publicado resultados positivos para la tinción de la MLI en la cirugía del agujero macular con otro colorante vital, el Brilliant Blue (21-23), que además parece tener un perfil de seguridad favorable (24). Aunque parece presentar ventajas con respecto al AT, este quedaría como una alternativa válida para la tinción de la MLI.

En resumen, los resultados de nuestras series sugieren que la tinción con AT a concentraciones de hasta el $0,15 \%$ son seguras para teñir la MLI, dado que no se ha detectado la presencia de daño funcional mediante ERG. Además, se obtienen mejorías significativas de la AV tanto a altas como a bajas concentraciones.

\section{BIBLIOGRAFÍA}

1. Mester $V$, Kuhn F. Internal limiting membrane removal in the management of full-thickness macular holes. Am J Ophthalmol 2000; 129: 769-777.

2. Brooks HL. Macular hole surgery with and without internal limiting membrane peeling. Ophtahlmology 2000; 107: 1939-1949.

3. Ben Simon GJ, Desatnik H, Alhalel A, Treister G, Moisseiev J. Retrospective analysis of vitrectomy with and without internal limiting membrane peeling for stage 3 and 4 macular hole. Ophthalmic Surg Lasers Imaging 2004; 35: 109-115.

4. Burk SE, Da Mata AP, Snyder ME, Rosa RH Jr, Foster $R E$. Indocyanine green-assisted peeling of the retinal internal limiting membrane. Ophthalmology 2000; 107: 2010-2014.

5. Da Mata AP, Burk SE, Foster RE, Riemann CD, Petersen $M R$, Nehemy $M B$, et al. Long-term follow-up of indocyanine green-assisted peeling of the retinal internal limiting membrane during vitrectomy surgery for idiopathic macular hole repair. Ophthalmology 2004; 111: 22462253.

6. Gandorfer A, Haritoglou C, Gass CA, Ulbig MW, Kampik A. Indocyanine green-assisted peeling of the internal limiting membrane may cause retinal damage. Am J Ophthalmol 2001; 132: 431-433.

7. Engelbrecht NE, Freeman J, Sternberg P Jr, Aaberg TM $S r$, Aaberg TM Jr, Martin DF, et al. Retinal pigment epithelial changes after macular hole surgery with indocyanine green-assisted internal limiting membrane peeling. Am J Ophthalmol 2002; 133: 89-94.

8. Haritoglou C, Gandofer A, Gass CA, Schaumberger M, Ulbig MW, Kampik A. Indocyanine green-assisted peeling of the internal limiting membrane in macular hole surgery affects visual outcome: a clinicopathologic correlation. Am J Ophthalmol 2002; 134: 836-841.

9. Kanda S, Uemura A, Yamashita T, Kita H, Yamakiri K, Sakamoto T. Visual field defects after intravitreous administration of indocyanine green in macular hole surgery. Arch Ophthalmol 2004; 122: 11447-11451.

10. Ando F, Yasui O, Hirose H, Ohba N. Optic nerve atrophy after vitrectomy with indocyanine green-assisted internal limiting membrane peeling in diffuse diabetic macular edema. Graefe's Arch Clin Exp Ophthalmol 2004; 242: 995-999.

11. Perrier M, Sebag M. Trypan blue-assisted ILM peeling of the internal limiting membrane during macular hole surgery. Am J Ophthalmol 2003; 110: 903-905.

12. Haritoglou C, Eibl K, Schaumberger M, Mueller AJ, Priglinger $S$, Alge $C$, et al. Functional outcome after trypan blue-assisted vitrectomy for macular pucker: a prospective, randomized, comparative trial. Am J Ophthalmol 2004; 138:1-5.

13. Haritoglou C, Gandorfer A, Schaumberger M, Priglinger $S G$, Mueller AJ, Gass CA, et al. Trypan blue in macular pucker surgery: an evaluation of histology and functional outcome. Retina 2004; 24: 582-590.

14. Narayanan R, Kenney MC, Kamjoo S, Trinh TH, Seigel $G M$, Resende GP, et al. Trypan blue: effect on retinal pigment epithelial and neurosensory retinal cells. Invest Ophthalmol Vis Sci 2005; 46: 304-309. 
15. Rebolleda G, Muñoz-Negrete FJ, Suarez-Figueroa M. Trypan blue staining in vitreoretinal surgery. Ophthalmology 2004; 11: 1622-1623.

16. Ezra E, Gregor ZJ. Surgery for idiopathic full-thickness macular hole. Two-year results of a randomized clinical trial comparing natural history, vitrectomy, and vitrectomy plus autologous serum: Moorfields macular hole study group report no. 1. Arch Ophthalmol 2004; 122: 224-236.

17. Marmor MF, Zrenner E. Standard for clinical electroretinography (1999 update). Doc Ophthalmol 1999; 97:143156.

18. Gale JS, Proulx AA, Gonder JR, Mao AJ, Hutnik CM. Comparison of the in vitro toxicity of indocyanine green to that of trypan blue in human retinal pigment epithelium cell cultures. Am J Ophthalmol 2004; 138: 64-69.

19. Veckeneer M, Van Overdam K, Monzer J, Kobuch K, van Marle W, Spekreijse H, et al. Ocular toxicity study of trypan blue injected into the vitreous cavity of rabbit eyes. Graefe's Arch Clin Exp Ophthalmol 2001; 239: 698-704.

20. Balayre S, Boisonnot M, Paquereau J, Digiero P. Evalua- tion of trypan blue toxicity in idiopathic epiretinal membrana surgery with macular function test using multifocal electroretinography: seven prospective case studies. J Fr Ophtalmol 2005; 28: 169-176.

21. Remy M, Thaler S, Schumann RG, May CA, Fiedorowicz $M$, Schuettauf $F$, et al. An in vivo evaluation of Brilliant Blue $G$ in animals and humans. Br J Ophthalmol 2008; 92: 1142-1147.

22. Enaida H, Hisatomi T, Hata Y, Ueno A, Goto Y, Yamada $T$, et al. Brilliant blue $G$ selectively stains the internal limiting membrane/brilliant blue G-assisted membrane peeling. Retina 2006; 26: 631-636.

23. Mochizuki Y, Enaida H, Hisatomi T, Hata Y, Miura M, Arita $R$, et al. The internal limiting membrane peeling with brilliant blue $G$ staining for retinal detachment due to macular hole in high myopia. Br J Ophthalmol 2008; 92:1009.

24. Enaida H, Hisatomi T, Goto $Y$, Hata $Y$, Ueno A, Miura M, et al. Preclinical investigation of internal limiting membrane staining and peeling using intravitreal brilliant blue G. Retina 2006; 26: 623-630. 